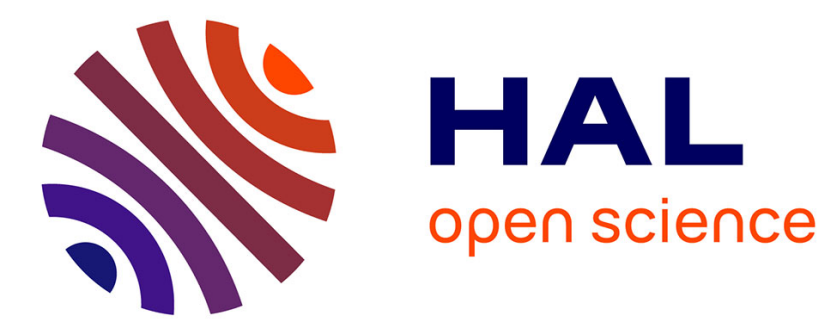

\title{
L'avenir de la télévision ? Régimes d'immersion et fantasme de communauté \\ Yves Citton
}

\section{To cite this version:}

Yves Citton. L'avenir de la télévision ? Régimes d'immersion et fantasme de communauté. Multitudes, 2010, pp.203-213. hal-00846491

\section{HAL Id: hal-00846491 \\ https://hal.science/hal-00846491}

Submitted on 24 Jul 2013

HAL is a multi-disciplinary open access archive for the deposit and dissemination of scientific research documents, whether they are published or not. The documents may come from teaching and research institutions in France or abroad, or from public or private research centers.
L'archive ouverte pluridisciplinaire HAL, est destinée au dépôt et à la diffusion de documents scientifiques de niveau recherche, publiés ou non, émanant des établissements d'enseignement et de recherche français ou étrangers, des laboratoires publics ou privés. 


\section{Lavenir de la télévision? Régimes d'immersion et fantasme de communauté Yves Citton}

La télévision est un fantasme. Au sens étymologique du mot phantasma: une apparition, une vision, une image, un ectoplasme, un fantôme. Par la magie des ondes et des flux électriques, des choses et des gens (qui ne sont vraiment ni des choses ni des gens) apparaissent chez moi, dans mon espace privé. Lars von Trier, Thom Yorke, Cate Blanchett, le Président, Daniel Cohn-Bendit viennent dans mon salon pour discuter de leur dernier film, de leur prochain album, de leur carrière, ou de l'avenir du pays. Chez moi, sans que j'aie besoin ni de me déplacer, ni de faire la queue, ni d'acheter un billet. Dorénavant, ils viennent même à l'heure qui me convient (et sans publicité). Et bientôt, ils pourront se promener avec moi, à la campagne, dans la rue, dans le métro. La télévision est un énorme fantasme, multiforme et en voie d'ubiquité.

Dans ces conditions, comment l'avenir de la télévision pourrait-il ne pas relever lui aussi du fantasme, de multiples fantasmes? C'est l'un de ces fantasmes qu'on va explorer ci-dessous - celui qui projette la télévision dans un avenir où elle est vouée à se dissoudre, à s'évaporer, comme un ectoplasme, comme le fantôme qu'elle est.

\section{Trois régimes d'immersion}

Il convient pour commencer de distinguer au moins trois régimes d'immersion. On peut tout d'abord se plonger dans une bulle sensorielle comme on plonge dans une piscine. Tout ce que vous voyez, entendez, percevez, appartient à cette bulle close sur elle-même, où que vous tourniez votre attention. C'est ce mode d'immersion que visent les différents dispositifs de grande taille proposés comme des expériences sensorielles totales, de l'Imax aux projections à $360^{\circ}$ et aux lunettes virtuelles. Appelons cela l'immersion globale. L'idéal en serait de nous plonger dans un univers artificiel, modulable à volonté, qui nous entou- 
re d'images sans bord, de sons et d'odeurs paraissant émaner de ces images elles-mêmes. Le défi est de parvenir à reproduire au mieux toute la largeur de notre champ perceptif ${ }^{1}$. Ce type de dispositif nous emprisonne aujourd'hui physiquement dans un lieu très limité du réel, dans lequel l'irréel parvient à être produit artificiellement avec la plus grande difficulté technique mais avec le plus grand réalisme. Le spectateur est généralement assis dans son fauteuil; au mieux il peut tourner la tête; dans les cas les plus poussés, il est debout et peut se déplacer, mais toujours à l'intérieur d'un espace clos. En pratique, l'immersion globale est donc toujours imparfaite, en ce que le bord de cet univers sensoriel reste toujours perceptible, et que je peux toujours déplacer mon attention sur lui afin de m'extirper du lieu où j'accepte volontairement de me plonger. Le fauteuil sur lequel je suis assis, la tête de mon voisin de devant, l'indicateur de sortie de secours toujours allumé, l'écran lui-même contre lequel je finirai par buter si des lunettes 3D me font confondre les images avec une réalité actuelle: tout cela trahitl'état d'immersion artificiel dans lequel je me trouve.

On peut aussi, alternativement, se plonger dans un branchement ponctuel qui nous accompagne partout où l'on va, comme un voyageur dans un pays chaud porte avec lui un spray rafraîchissant dont il peut s'asperger en tout lieu. Vous êtes libre de vous déplacer à votre gré dans l'espace sans bord de la réalité sensorielle commune, mais vous pouvez en tout endroit vous connecter à une lucarne qui vous donne accès à un univers sensoriel différent. C'est le Blackberry, l'I-phone et autre I-pad que vous portez toujours sur vous, et qui vous permet, où que vous soyez, de visionner Avatar de regarder votre série TV favorite, de vérifier en temps réel l'état de votre jardin ou de vos investissements boursiers. Appelons cela l'immersion ubiquitaire ${ }^{2}$. L'idéal, dont on n'est guère éloigné, serait de fournir à ce genre d'écrans portables une couverture globale étendue à l'ensemble de la planète, sans rupture de réseau.

Ce type de dispositif nous laisse libres de nos déplacements dans l'espace, mais nous contraint à focaliser notre attention sur une portion très limitée de notre champ sensoriel. Je peux marcher, rouler, voler où je le souhaite, mais mes yeux doivent rester fixés sur un écran de petite taille. La faiblesse sensorielle de tels dispositifs vient de ce que la plus grande partie de mon champ perceptif est occupé par le bord - par tout ce que je peux voir et entendre autour de mon petit écran, par les secousses que subit mon siège au moment d'un atterrissage, par mes voisins de train qui parlent trop bruyamment ou qui lâchent un pet. Contrairement à l'immersion globale, dans laquelle je suis passivement plongé, et dont seuls des points de détails trahissent l'irréalité, l'immersion ubiquitaire demande au spectateur de se plonger activement en elle, par un effort d'attention toujours sourdement en lutte avec tout ce qui, du bord, menace sans cesse de le distraire.

On peut enfin entrevoir désormais un troisième régime d'immersion alliant les deux modes précédents en nous plongeant dans une «réalité augmentée», qui

1 Pour une élaboration théorique des enjeux des notions d'art immersifet d' intelligence immersive, très éclairante pour le régime de «l'immersion globale», voir Joseph Nechvatal, Towards an Immersive Intelligence. Essays on the Work of Art in the Age of Computer Technology and Virtual Reality (1993-2006), New York, Edgewise, 2009, p. 24-39. (Les thèses de Nechvatal sont présentées dans ma contribution en anglais à ce numéro de Multitudes.)

Sur la notion d'ubiquité prise dans ce sens, voir Adam Grienfield, Everyware. La révolution de l'ubimédia, Paris, FYP Editions, 2007 tout à la fois nous entoure complètement et nous suit partout où nous allons, comme ces personnages malchanceux de dessins animés que suit un nuage de pluie partout où ils se trouvent, même en plein été. En portant des «lunettes virtuelles» (dotées d'écouteurs, voire d'un petit mécanisme de spray olfactif) connectées à Internet, on pourra bientôt simultanément percevoir la réalité qui nous entoure et la supplémenter d'informations ou de sensations artificiellement produites par la machine miniaturisée, modulables à l'envi, adaptées en temps réel à notre environnement actuel. On peut parler dans ce cas d'immersion microcosmique, dans la mesure où le dispositif virtuel génère un microcosme qui nous accompagne où que nous allions, à l'image d'un microclimat portatif. Si je suis perdu dans une ville, je fais afficher le nom des rues où je me trouve. Si la lumière est insuffisante, j'enclenche la vision nocturne par infra-rouge. Si mon voisin parle trop fort, je monte le son de mon téléphone ou de ma musique. S’il sent mauvais, j'active un parfum qui masque son odeur.

Ce troisième type de dispositif ne connaît plus de bord, il recouvre toute la largeur de mon champ perceptif, puisque c'est dans la réalité elle-même que j'évolue, et que l'appareillage d'augmentation agit directement sur mes organes de perception. Il ne m'emprisonne plus dans aucun lieu d'artificialité (comme la salle du Imax), puisque je peux le porter avec moi où que j'aille. Alors que dans les dispositifs précédents s'opérait un basculement discontinu, brutal et binaire, sur les points servant de bord, entre la réalité et l'image, entre le réel et l'irréel, entre l'actuel et le virtuel, on peut ici moduler de façon continue le passage progressif entre une réalité non augmentée (j'éteins l'appareil) et un univers totalement fictif (je me projette Avatar, version olfactisée), en passant par toute la gamme des corrections ponctuelles imaginables (j'augmente le volume de la voix de mon interlocuteur s'il parle trop bas, j'active une odeur de croissant, je repeins le vert en jaune dans le paysage que je regarde, j'efface tous les individus cravatés de mon champ de vision).

\section{Un affaiblissement progressif
de l'immersion globale}

Si l'histoire de la télévision, quoiqu'encore très jeune, a déjà connu des transformations significatives quant à son régime d'immersion, notre conscience de ces transformations a pris un retard considérable sur leurs évolutions réelles. Nous raisonnons parfois sur la télévision comme s'il s'agissait simplement du «petit écran» des années 1950, que les miracles de l'Internet parviendraient seulement aujourd'hui à libérer de sa boîte originelle pour la rendre enfin portative. En survolant cette histoire du point de vue des différents régimes d'immersion, on voit toutefois se succéder plusieurs phases bien distinctes, dans lesquelles les effets des innovations technologiques vont rarement dans la direction originellement attendue.

Le premier type d'évolution, celui qui concerne le régime de l'immersion globale, n'est guère intéressant. Depuis les cinquante lignes des premiers appareils télévisuels jusqu'à la haute définition actuelle, du souffle des premières émissions jusqu'au son digital des transmissions câblées, du noir-blanc à la couleur et jusqu'à la 3D, depuis le «petit écran» originel vers les écrans géants actuels, la télévision est restée un média 
remarquablement faible du point de vue d'une immersion sensorielle globale. On paraît avoir assisté en réalité à une double évolution, apparemment contradictoire, qui tire la télévision en deux sens contraires.

D’une part, les dispositifs techniques tendent à augmenter le «réalisme» des images projetées, ainsi que la largeur du champ couvert, participant d'une augmentation à la fois intensive et extensive de l'expérience audio-visuelle. Force est toutefois de reconnaitre qu'en comparaison avec une projection en Imax, même le plus géant des écrans - à moins d'avoir le nez collé sur lui au milieu d'une chaîne hifi quadriphonique - relève d'une immersion très imparfaite. Quel que soit le modèle qu'on achète une TV reste un petit écran, clairement délimité par des effets de bord omniprésents. Comme le remarquait John Berger dans Ways of Seeing ${ }^{3}$, la spécificité de la télévision est de permettre le découpage d'un fragment d'image du monde - il prenait l'exemple d'un tableau de Botticelli - pour l'insérer dans notre salon, sur fond de la tapisserie de notre mur, à côté de notre fenêtre ouverte, et surmonté d'une photo de nos enfants souriant en caleçons de bain sur une plage espagnole. Différents avertissements et différentes légendes ont poussé les téléspectateurs à «laisser une lampe allumée dans la chambre» pour prévenir les effets supposés néfastes d'une concentration exclusive sur une boîte magique restée toujours vaguement inquiétante quant à ses effets insoupçonnés - avec pour effet de réduire considérablement la force d'immersion, contrairement à l'obscurité complète que cherchent à produire tous les dispositifs d'immersion globale.

En même temps que les dispositifs techniques augmentaient (marginalement) l'intensité et l'extension de l'immersion perceptive, les conditions de consommation des produits télévisuels tendaient au contraire à diminuer les effets d'immersion. À mesure que la télévision a vieilli, on a prêté une attention toujours plus distraite et superficielle à ce qui sort d'une boîte devenue de moins en moins magique. En 1960, la famille réunie religieusement devant l'émission nocturne (et fascinée par la nouveauté du procédé) pouvait sans doute se sentir profondément immergée dans le programme diffusé, sur le modèle des premiers spectateurs de l'invention des frères Lumière, dont la légende veut qu'ils aient eu peur d'être effectivement écrasés par le train qui arrivait en gare de La Ciotat. À l'inverse, on sait à quel point il est difficile de mesurer aujourd'hui l'audience réelle d'une émission, puisque la plupart d'entre nous (ne) «regardent» (pas) la télévision en faisant le repassage, en parcourant un magazine, voire en passant l'aspirateur (ce qui reste la meilleure façon de consommer CNN).

Dans un dispositif où les effets de bords sont aussi invasifs, c'est bien plus de l'intensité d'attention que de la qualité technique des images que dépend l'immersion. Cette intensité d'attention n'est bien entendu nullement constante, variant selon les ménages et selon les moments entre une valeur minimale durant les pauses publicitaires, une valeur moyenne au moment des informations, et quelques pics lors des penaltys d'une finale de la coupe du monde ou du dernier épisode d'une série populaire. Contrairement aux fausses évidences d'un déterminisme technologique conçu de façon trop étroite, c'est donc plutôt une diminution de l'effet d'immersion globale qu'a connue l'histoire de la télévision depuis sa première apparition.

3 John Berger, Ways of Seeing, 1972, BBC Series (disponible par tranches sur YouTube). Voir ici même l'article que Paula Roush consacre à cette série.

\section{Une ubiquité raréfiée}

L'immersion par ubiquité a, elle aussi, été traversée de deux mouvements contradictoires. On a sans doute raison de faire de la télévision comme telle un dispositif de communication de plus en plus ubiquitaire au fur et à mesure de l'avancée du XX siècle. D’un poste trônant au milieu du salon, on est passé à une télévision dans chaque chambre, puis désormais à une télévision portative, qu'on pourra bientôt regarder dans le métro, sur la plage ou durant l'ascension d'un sommet alpin. Nous voilà donc de plus en plus immergés dans un médium de plus en plus ubiquitaire, que chacun peut désormais « regarder» sans interruption, de son lever à son coucher, où qu'il aille et quoi qu'il fasse: on a déjà des statistiques sur le nombre d'accidents dus au fait que les conducteurs regardaient la télévision (ou surfaient sur Internet). La relative faiblesse de l'attention consacrée à la télévision actuelle serait donc (plus que) compensée par son ubiquité potentiellement absolue.

Selon le modèle du synopticon décrit il y a une quinzaine d'années par Thomas Mathiesen (en symétrie inverse du panopticon benthamo-foucaldien), nous nous dirigeons donc vers une société de contrôle dans laquelle la direction du regard impliqué dans la «surveillance» se serait retournée. L'important ne serait plus tant (ou plus seulement) la surveillance exercée depuis les centres de pouvoir (le gardien au milieu du cercle) sur les multitudes environnantes (les prisonniers dans leur cellule); les mécanismes de contrôle reposeraient de plus en plus sur le fait que les multitudes ont toutes le regard fixé sur un point central (quelques stars, quelques scandales, quelques divertissements populaires).

Pour Mathiesen, la télévision représente le modèle dominant du synopticon et des nouveaux médias à travers lesquels «les multitudes regardent les élites» ( «the many see the few»). L'ubiquité des écrans portables signifie donc l'intensification du synopticon: les yeux des many peuvent désormais être rivés sur les few durant chaque seconde de leur journée, transformant par là-même leur vie en un interminable rêve (jamais véritablement) éveillé. Une telle situation, bien entendu, inquiète Mathiesen: «le synopticisme, à travers les mass médias en général et la télévision en particulier, a pour principal effet de diriger et contrôler, ou de discipliner, nos consciences $»^{4}$. L'ubiquité accrue, que rendent possible les nouveaux développements technologiques, se traduirait donc par une puissance de contrôle elle aussi accrue.

On sait que les choses sont moins simples que cela. L'autre transformation majeure du paysage télévisuel, déjà largement effectuée au cours des 30 dernières années, consiste en la multiplication spectaculaire des chaînes entre lesquelles les téléspectateurs peuvent zapper. Or, ici aussi, les effets de la multiplication de l'offre vont dans le sens inverse des effets induits par le développement technologique. L'ORTF ou la BBC bénéficiaient d'une ubiquité bien supérieure à celle de n'importe quelle chaîne actuelle. De ce point de vue, les téléspectateurs actuels sont bien moins immergés dans un univers perceptif en charge de discipliner leur conscience: même s'ils restent plus constamment connectés à leur machine télévisuelle, le contrôle se relâche dès lors $\left(1^{\circ}\right)$ qu'ils peuvent choisir de se brancher sur telle source de programme plutôt que sur telle autre, dès lors $\left(2^{\circ}\right)$ que le nombre de ces sources augmente au point de se compter par centaines

4 Thomas Mathiesen, "The Viewer Society: Michel Foucault's Panopticon Revisited", Theoretical Criminology, vol. 1: 2 (1997), p. 230 . 
et bientôt par milliers (voire par millions, dès lors que chacun peut diffuser sur YouTube ses petits programmes faits maison), et dès lors $\left(3^{\circ}\right)$ que le téléspectateur n'est pas condamné à choisir une seule source de façon stable, mais peut à chaque instant en changer par le zapping, regarder telle chaîne le matin et telle autre chaîne le soir, voir diviser son écran en trois pour «regarder» trois émissions en même temps.

Sous ces trois conditions, l'ubiquité croissante des appareils télévisuels s'est donc accompagnée d'une raréfaction croissante des audiences qui remet en question le concept même d'immersion. On est toujours immergé dans «quelque chose», et ce quelque chose doit être doté d'une certaine unité pour qu'on puisse véritablement parler d'immersion. Le dispositif télévisuel est un médium plutôt qu'un milieu. Parlerait-on d'apprendre un langage "par immersion» si j'allais passer une année dans une maison internationale dont chaque occupant parlerait une langue différente? Soit nous partagerions une même langue seconde (disons l'anglais), et je me plongerais alor dans un milieu anglais. Soit j’identifierais les quelques personnes qui pratiquent des langues dans lesquelles je peux me débrouiller (disons l'italien et l'allemand), et cette double immersion pourrait me permettre d'améliorer ma maîtrise des deux médiums simultanément. Soit les participants auraient été soigneusement choisis de façon à ce que personne ne parle la langue d'aucun autre, et il nous faudrait alors apprendre à développer des langues communes et des modes de traduction - mais il s'agirait dans ce cas de tout autre chose que d' "apprendre une langue par immersion».

\section{Modèle caméléonesque et modèle multipolaire}

N'est-ce pas dans une situation partiellement similaire que se trouve le téléspectateur auquel s'offrent des centaines de chaînes différentes? L'analogie avec la maison internationale nous aide à répondre à cette question. On peut considérer que la multiplication des chaînes ne suffit nullement, en soi, à garantir une diversification de l'offre, et l'on est alors dans le cas de l'immersion dans l'anglais mondialisé. Quelle que soit la personne à laquelle je parle (de quelque pays qu'elle vienne), c'est toujours (en gros) le même idiome (impur) que je parlerai dans cette maison apparemment internationale, mais en réalité anglophone. Quelle que soit la chaîne que je regarde, c'est toujours (plus ou moins) la même soupe qu'on me sert (avec ses doses stables de publicité, de sujets à la mode et de manies du moment), au sein d'un paysage audiovisuel qui ne multiplie les entités que pour mieux assurer la permanence du même.

C'est ici le modèle caméléonesque de la médiasphère capitaliste: la quête de profit poussant chacun à vouloir capter le goût des plus larges audiences, puisque ce sont elles qui garantissent l'afflux des ressources publicitaires, chacun tente d'imiter "ce qui marche», et tout le monde se trouve changer périodiquement de couleur en fonction de celle qui paraît être au goût du jour. Un tel comportement est bien une affaire de milieu, et on est effectivement alors dans un régime d'immersion, que l'ubiquité des machines de branchement rend sans doute plus inquiétant encore

On peut toutefois imaginer un autre modèle, correspondant à la deuxième hypothèse de la maison internationale, celle dans laquelle je passe mon année à parler allemand et italien avec quelques-uns des habitants, sans jamais vraiment converser avec les autres. Nos branchements médiatiques paraissent en effet se concentrer sur deux ou trois chaînes auxquelles nous revenons régulièrement, à l'exclusion quasi-totale de centaines d'autres. On est ici dans un modèle multipolaire: la multiplication des chaînes introduit un jeu limité dans la machine médiatique, qui nous met à égale distance entre l'ubiquité effective de l'ORTF et la raréfaction des audiences due à la multiplication de l'offre. Ce modèle multipolaire participe toujours d'une immersion, puisque je tends à me baigner chaque matin et chaque soir dans les mêmes rivières (où je me retrouve nager généralement avec bon nombre de mes semblables), même si les flots, la couleur, l'odeur et le goût de chacune de ces rivières peuvent différer sensiblement de l'une à l'autre.

Du point de vue des dispositifs de contrôle, ce modèle présente des caractéristiques intéressantes. En première approche, on pourrait le considérer comme moins terrifiant que l'univers caméléonesque: on échappe au cauchemar consensuel où les individus se comportent à l'unisson, comme des bancs de poissons ou des armées de clones. On les voit s'organiser en troupeaux aux couleurs variées, toujours susceptibles de devenir des troupes mobilisées autour de causes conflictuelles. La logique synopticiste est enrayée: même si la tendance des many est toujours de regarder les few, les disciplines se pluralisent et les contrôles s'atténuent, dès lors qu'on a affaire à many $f e w$, à plusieurs centres d'attention en compétition les uns avec les autres, dont chacun mobilise ses partisans avec des agendas relativement différenciés.

On sait toutefois que rien n'assure mieux la stabilité d'un système que d'y introduire un peu de pluralité et de diversité. Pour peu qu'un excentrique obtienne un succès inattendu auprès de ses semblables, une société de caméléons peut basculer soudainement dans les comportements les plus imprévisibles et les plus extrêmes. Les esprits critiques auront donc sans doute raison de considérer le modèle multipolaire (et l'accueil bienveillant de certaines alternatives critiques) comme un mécanisme de défense par lequel un système d'hégémonie globale prévient des changements plus radicaux et plus menaçants pour sa stabilité dans le long terme.

\section{Le fantasme de la dissolution microcosmique}

Le troisième cas envisagé dans la maison internationale est toutefois le plus intéressant. Dans quoi y aurait-il immersion, si je passais une année dans un espace habité par des gens avec lesquels je ne partage aucune langue commune? Cette situation ressemble à celle qu'on nous dépeint comme constituant l'avenir de la télévision, à l'horizon d'une fragmentation des médias majoritaires de masse. Elle correspond aussi au régime de l'immersion microcosmique évoqué au début de cet article.

Imaginons que chacun puisse être branché en permanence sur sa télévision-ordinateur-écouteurs-lunettes-virtuelles (avec spray et microphone incorporé, pour donner ses ordres à la machine) - appelons l'ensemble du dispositif son « Portable». Imaginons aussi que chacun ait à sa disposition, en tous lieux, non seulement des millions d'émissions disponibles sur demande, pour les moments où il n'y a rien d'intéressant à percevoir autour de lui, mais aussi une vaste palette de transformations lui permettant de corriger les données qui lui parviennent de son environnement immédiat. Chacun ne tendra-t-il pas dans ce cas à s'enfoncer progressivement dans «son 
monde propre»? Ne lui sera-t-il pas de plus en plus difficile de «partager» ce monde avec ceux qui voient, entendent et sentent une «réalité» différente de la sienne?

Mon Portable inclura bien entendu une fonction de traduction automatique, ce qui résoudra les problèmes de communication au sein de la maison internationale. Il me suffira de sélectionner la langue de mon interlocuteur pour que ses propos soient aussitôt compréhensibles dans ma langue propre. La question est toutefois de savoir jusqu'à quel point cette langue me sera "propre», et jusqu'à quel point ses propos me seront «compréhensibles». Je pourrai choisir précisément que type de free jazz (New York Downtown scene des années 1980), de conférences (cours de Deleuze à Vincennes), de livres audio (Diderot), de films (les frères Dardenne), d'émissions radiophoniques (Zazirocratie), de programmes télévisés (Ways of Seeing) je sélectionne pour peupler mon univers mental; je pourrai choisir tout aussi précisément mes sources d'informations préférées, ainsi que mes contenus pertinents (grâce à des agrégateurs de flux taillant les nouvelles quotidiennes à mes intérêts et à mes goûts propres); je pourrai également choisir avec une précision merveilleuse quels types de données sensorielles je sélectionne dans mon environnement (neutraliser par avance les odeurs qui me soulèvent le cœur, redessiner les vêtements de femmes pour dénuder leurs épaules, effacer les discussions sportives entre voisins de métro qui me distraient quand j'essaie de me concentrer): dans de telles conditions, ne vais-je pas progressivement me creuser un monde tellement «propre» que de larges pans de ce qui fait la réalité d'autrui (les sports, Desperate Housewives, Mozart, Télé Matin, la viande, le Parti Socialiste, Sonny Rollins, Bollywood, la littérature contemporaine, Michael Jackson) ne m'apparaîtront que comme des «saletés» à éliminer - et donc ne m’apparaîtront plus. Les différences entre les langues ethniques, parlées par les communautés humaines jusqu'à aujourd'hui, n'auront été surmontées, grâce au traducteur automatique inclus dans mon Portable, que pour engendrer des différences encore plus difficilement surmontables, entre des individus de plus en plus idiotiquement singularisés.

Les évolutions des trois régimes d'immersion présentées sommairement ci-dessus semblent pointer dans une même direction, où prend forme un fantasme apocalyptique de dissolution sociale. Retraçons-les brièvement pour prendre la mesure de ce fantasme, qui commence à hanter de plus en plus de nos contemporains : 1) Malgré le réalisme à toujours plus haute définition d'écrans toujours plus géants, le téléspectateur est de moins en moins immergé (globalement) dans des programmes qu'il ne regarde plus que d'un œil distrait. 2) Malgré le fait que nous puissions désormais rester l'œil collé à un écran devenu ubiquitaire, où que nous allions et du matin au soir, la multiplication des programmes qui nous sont offerts a pour effet que nous ne sommes plus immergés dans une sphère médiatique commune, mais dans une multitude de bulles de plus en plus personnalisées. 3) Alors que le développement technologique du Portable paraissait devoir ouvrir la perspective d'une immersion totale, sans plus aucun bord, instaurant une continuité parfaite entre le réel et le virtuel, effaçant par avance toute cause possible de distraction, garantissant une totale liberté de mouvement en tout point de la planète - l'immersion microcosmique paraît au contraire menacer de dissoudre tout monde commun. Loin de nous plonger dans un milieu définitivement homogénéisé, la dissolution microcosmique récuserait la notion même d'immersion, chacun se trouvant désormais incapable de voir ou de dire dans quoi il est plongé. Si un enfermement s'esquisse à l'horizon de cet avenir, ce ne sera plus dans le milieu que j'habite, mais dans la bulle d'idiotie que je suis.

\section{L'avenir du synopticisme}

Dire que cet avenir de dissolution relève du fantasme ne revient pas à exclure la possibilité de sa réalisation. C'est seulement le remettre à sa place, qui est, comme tout ce qui sort de la télévision elle-même, une apparition, un fantôme. Sa vertu lui vient moins de ce qu'il corresponde (ou non) à une réalité à venir, que de ce qu'il aide à faire apparaitre dans notre présent - et qui tient à deux choses.

D'une part, ce fantasme de dissolution microcosmique nous pousse à mettre la lumière sur les nécessités de collaboration fonctionnelle imposées par la production matérielle de l'immatériel. Poussé par un désir de vivre dans un monde débarrassé de tout stress, je pourrai sans doute programmer mon Portable à effacer de mon champ de vision tout ce qui ressemblera à une facture. Il suffira toutefois de quelques semaines pour me ramener à la triste et pauvre réalité (bien en mal d'augmentation), dès lors que les fournisseurs d'électricité ou de connexion Internet auront interrompu leurs flux d'énergie et de données. De même ne suffit-il pas de transmorpher des cailloux en beignets pour se régaler de pâtisserie: en jouant avec ce type-là de virtuel, on risque surtout de se casser les dents et de mourir de faim.

On peut donc suspecter le fantasme de la dissolution microcosmique d'être leurrant en ce qu'il méconnaît qu'une certaine forme de commun est la pré-condition de la vie individuelle, avant d'être le produit de la collaboration entre les individus. Le fantasme de l'isolation dans la bulle d'idiotie oublie que l'on est toujours-déjà immergé dans du commun en acte, selon ce que Heidegger désignait comme notre Geworfenheit, exprimant par là le fait que nous sommes jetés-dans-le-monde avant tout choix d'y être ou non, d'y être situé ici plutôt que là, sous cette identité plutôt que sous telle autre.

Sans entretien des usines électriques et des serveurs, et des pièces de rechange nécessaires à les réparer, et des véhicules qui apportent ces pièces, et des fours qui font le pain que mangent les conducteurs de ces véhicules, et de tout l'ensemble des fonctions nécessaires à la production de la réalité et de ses augmentations virtuelles - sans ce commun préalable, il n'y a ni microcosme, ni donc risque de dissolution. Or cet ensemble fonctionnel impose ses exigences de collaboration réelle, qui assurent un minimum de fermeté à la reproduction du commun.

L'intérêt du fantasme de dissolution tient à ce qu'il nous rappelle que les exigences de collaboration effective ne s'imposent presque jamais directement, mais plutôt par le détour de constructions imaginaires, autrement dit de fantasmes (la volonté de Dieu, l'ordre du Père, la loi du Roi, le décret du Président, le vote du Peuple, les attentes du Public). Si, comme on le disait pour commencer, la télévision est un fantasme, c'est surtout à ce niveau - celui d'un fantasme de communauté - qu'il importe de mieux comprendre sa nature, sa fonction, sa puissance et sa fragilité.

Nous avons de la peine à reconnaître «la télévision» dans le dispositif de diffusion ubiquitaire des images et des sons (ainsi que des odeurs) que j’ai esquissé au 
titre de l'immersion microcosmique permise par les Portables. Non seulement on ne sait plus dans quoi on s'y trouverait immergé, mais cette dissolution relève d'un régime posttélévisuel. Félix Guattari décrivait déjà très précisément un tel régime, qu’il qualifiait de «postmédia» et dans la perspective duquel, en 1990, il trouvait des raisons d'espérer:

La jonction entre la télévision, la télématique et l'informatique est en train de s'opérer sous nos yeux et elle s'accomplira probablement durablement dans la décennie à venir. La digitalisation de l'image télé aboutit bientôt à ce que l'écran de télé soit en même temps celui de l'ordinateur et celui du récepteur télématique. Ains des pratiques aujourd'hui séparées trouveront-elles leur articulation. Et des attitudes, aujourd'hui de passivité, seront peut-être amenées à évoluer. Le câblage et le satellite nous permettront de zapper entre 50 chaînes, tandis que la télématique nous donnera accès à un nombre indéfini de banques d'images et de données cognitives. Le caractère de suggestion, voire d'hypnotisme, du rapport actuel à la télé ira en s'estompant. On peut espérer, à partir de là, que s'opérera un remaniement du pouvoir mass-médiatique qui écrase la subjectivité contemporaine et une entrée vers une ère postmédi consistant en une réappropriation individuelle collective et un usage interactif des machines d'information, de communication, d'intelligence, d'art et de culture ${ }^{5}$.

Un regard rétrospectif depuis cet avenir fantasmé nous permet toutefois d'identifier plus précisément en quoi consistait alors «la télévision», telle que nous la connaissions jusqu'au début du XXI ${ }^{e}$ siècle. La télévision n'était pas un appareil de diffusion des images et des sons, mais une macro-machine de production d'un fantasme de communauté. Ce qui comptait, ce n'était pas seulement qu'elle me permette de recevoir Lars von Trier, Thom Yorke, Cate Blanchett, le Président ou Daniel Cohn-Bendit dans mon salon, à l'heure du repas. Ce qui constituait son pouvoir effectif, c'est que j'en recevais l'impression qu'une multitude de mes semblables recevaient eux aussi toutes ces célébrités dans leur salon, au même moment que moi. La télévision vivait en réalité d'un double direct: non seulement elle pouvait représenter en temps réel ce qui était en train de se passer en tout point de la planète (une finale de match de foot, une ville soumise au feu de l'ennemi ou à une inondation catastrophique). Tout aussi important était direct de la diffusion et de la réception synchrone au sein des masses ainsi constituées par les médias en autant de communautés.

Ce dans quoi nous immerge vraiment la télévision, c'est donc dans le fantasme d'une communauté. Comme aime à le rappeler Daniel Bougnoux, «communiquer, c'est avant tout être et mettre en commun». Le contenu ou l'information à transmettre ne viennent que bien loin derrière la première fonction «communiante» que doit assurer la communication ${ }^{6}$. C'est cette commun(icat)ion macrosociale que dissout l'immersion microcosmique, et c'est pour ça que nous avons de la peine à y reconnaître «la télévision». En passant du mode push, dans lequel «les médias distri-

5 Félix Guattari, «Vers une ère postmédia» paru originellement dans Terminal n 51 (1990), disponible sur http://multitudes.samizdat.net/Vers-une-ere-postmedia

6 Daniel Bougnoux, Introduction aux sciences de la communication, Paris, La Découverte, nouvelle éd. 2001, p. 74 et passim. buaient les mêmes programmes pour tous» en nous «faisant partager les nouvelles des autres», à un mode pull, dans lequel «chacun peut ignorer le monde des autres en ne traitant que les messages pertinents pour sa bulle $»^{7}$, on a déjà commencé depuis plusieurs années à tuer la télévision. Pour le meilleur sans doute, comme le suggérait Félix Guattari... Mais peut-être y avait-il aussi quelques bénéfices secondaires à tirer de ce fantasme de communauté.

La «télévision» s'avère du même coup très mal nommée, puisque ce qui compte, ce n'est pas tant la «vision de loin» (Fern-sehen), que la «vision avec», la vision synchronisée que rend bien plus adéquatement la notion de synopticisme. L'avenir de ce que nous avons appelé «la télévision » depuis plus d'un demi-siècle est donc lié au destin du synopticisme, dont il n'est d'ailleurs nullement évident qu'il soit voué à disparaitre prochainement ${ }^{8}$. Dans quoi nous immergerons-nous au cours des décennies à venir? Allons-nous évoluer vers un dispositif plus démocratique au sein duquel les many en arrivent à regarder les many (plutôt que les few) ? Parviendrons-nous à assurer la maintenance du commun en l'absence de la communion soudée par le synopticisme?

Comme le suggérait Félix Guattari, c'est peut-être dans le champ des pratiques artistiques que se joue un avenir postmédia, pour autant qu'elles apprennent à profiter de cette dissolution de la télévision - qui les excluait jusque-là de façon massive - pour retrouver une nouvelle visibilité sur les ruines du synopticisme télévisuel, grâce à « un usage interactif des machines d'information, de communication, d'intelligence, d'art et de culture»? 


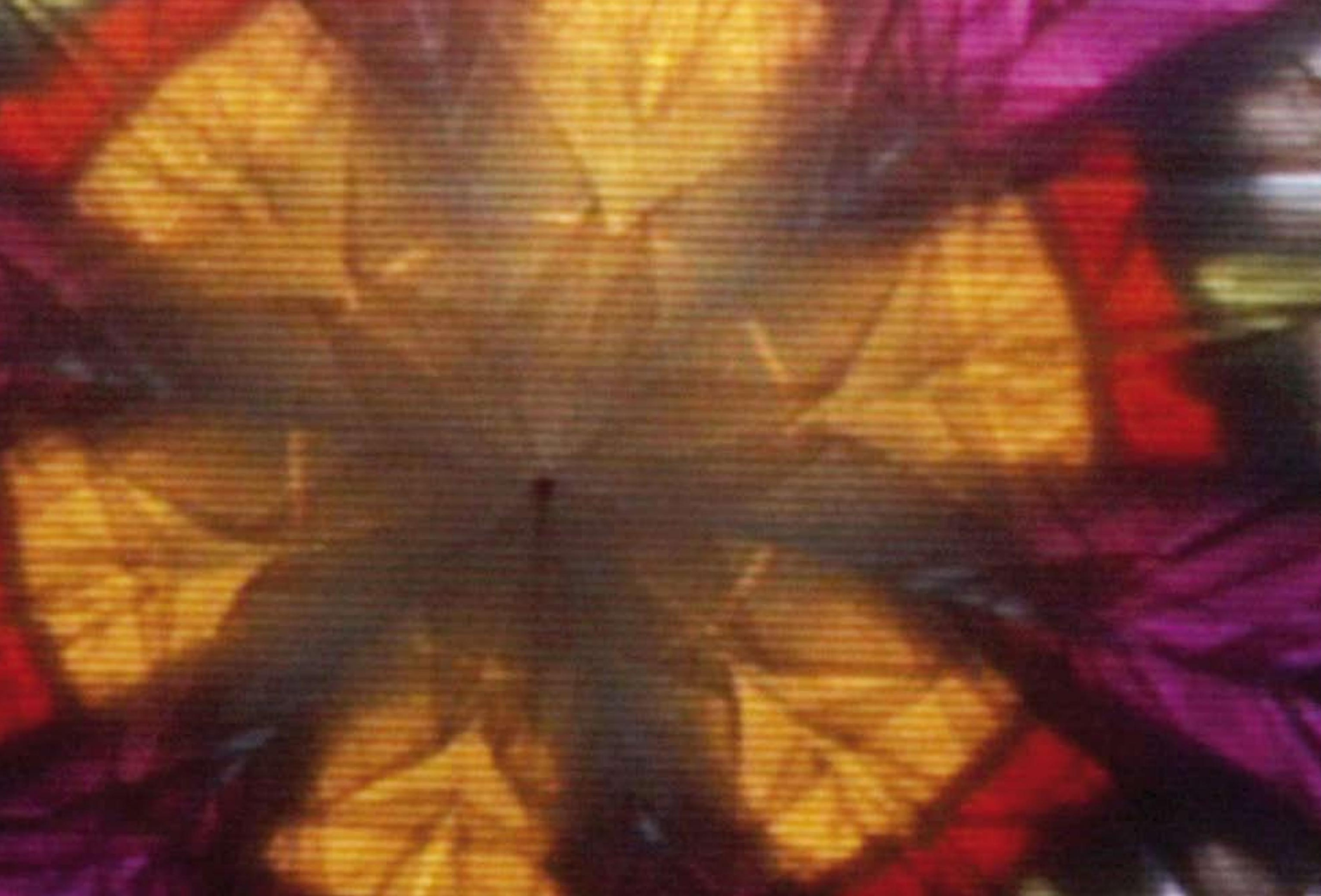

